American Journal of Infectious Diseases 5 (1): 31-39, 2009

ISSN 1553-6203

(C) 2009 Science Publications

\title{
Borderline Personality Symptoms and Human Immunodeficiency Virus Risk in Alcohol and Other Drug Abusing Adolescent Offenders
}

\author{
${ }^{1}$ Jessy G. Dévieux, ${ }^{1}$ Robert M. Malow, ${ }^{1}$ Rhonda Rosenberg, \\ ${ }^{1}$ Madhavan Nair, ${ }^{1}$ Deanne M. Samuels and ${ }^{2}$ Robert McMahon \\ ${ }^{1}$ Florida International University, Miami, Florida \\ ${ }^{2}$ University of Miami, Miami, Florida
}

\begin{abstract}
Problem statement: Incarcerated youth with borderline symptomatology represent a particularly at risk-population due to their enggement in risky behaviors. Five hundred twenty two adolescents were assessed for borderline symptomatology (MACI), engagement in risky behaviors and attitudes/knowledge. Approach:Adolescents were divided into two groups: low borderline (below the 60 scale score cutoff) and high borderline (subclinical and clinical range). Multivariate analyses were used to test for group differences. Results: The high borderline group had higher perceived susceptibility, greater knowledge, less favorable sexual and condom attitudes and less favorable behavioral intentions. There were no significant differences by group on sexual risk or substance use behaviors. A subset $(n=156)$ participating in a risk reduction experimental trial were followed three months post-intervention for differences in sexual risk and substance use. The high borderline experimental participants reported significantly more anal sex than the low borderline adolescents at 3 month follow-up. High borderline adolescents in the control group reported greater cocaine use than low borderline controls at 3 months, including trends suggesting more marijuana and alcohol use. At 3 month follow-up, no differences in cocaine, alcohol or marijuana use were detected between high and low borderline adolescents in the experimental group. Adolescents with higher borderline tendencies appear to realistically assess that they are at high risk of contracting HIV but may have less confidence in their ability to adopt HIV preventive behaviors. The results indicate that borderline personality symptoms may represent an important indicator of attitudes conducive to HIV transmission. Conclusion:Three-month follow-up data indicate the importance of examining borderline characteristics more microanalytically within research studies, including their potential role as moderators of intervention effect and their interactions with substance use, particularly cocaine. We discuss the further importance of matching this microanalysis with measures of the neurobiological dimension in order to further the field.
\end{abstract}

Key words: Borderline symptoms and HIV/AIDS, adolescent AOD and risky sexual behavior, borderline personality and AOD adolescent offenders, borderline personality, adolescents, HIV/AIDS, substance use and cocaine

\section{INTRODUCTION}

The co-occurrence of substance use and risky sexual behaviors has been well documented among adolescents and most particularly among incarcerated youth $^{[1-5]}$. Adolescents who also exhibit symptoms of personality disorders, specifically borderline personality, may be at even higher risk due to the synergistic effects of these disorders.

Among adolescents, borderline symptoms have been linked to high alcohol consumption and among those adolescents with borderline and alcohol use disorders, higher levels of impulsivity are observed compared to those without a substance use disorder ${ }^{[6,7]}$. The combination of the effects of substance abuse along with the impulsivity associated with borderlines may therefore further increase the risk of becoming infected with HIV due to substance-related cognitive impairment and risk behaviors (e.g., needle sharing, sex trade $)^{[8-10]}$. In addition, previous research has shown that individuals diagnosed with borderline personality initiate substance use at an earlier age, suggesting the likelihood of more severe outcomes due to substance use compared to those without the diagnosis ${ }^{[11,12]}$.

Corresponding Author: Robert Malow, Biscayne Bay Campus, C I-Suite 260, Florida International University, North Miami, Florida 33181-3600 Tel: 305.919.4222 
Among adolescents, those involved in the justice system represent a sub-sample of youth at very high risk for HIV. They initiate sex and drug use at an earlier age compared to the general population, use condoms less frequently during sex and have more permissive attitudes toward $\operatorname{sex}^{[13,14]}$. They have been shown to exhibit high levels of borderline personality features and sexual and drug use behaviors related to the transmission of $\mathrm{HIV}^{[15-17]}$. In addition, several investigators have reported high rates of psychiatric disorders, including borderline personality features in the adolescent offending population ${ }^{[18]}$.

Despite these findings and documentation of Axis II psychiatric symptoms being significantly associated with high risk sexual behavior among adolescents ${ }^{[19]}$, few studies have examined the association between borderline personality symptoms and HIV risk among Alcohol and Other Drug (AOD) abusing adolescents, particularly those involved with the criminal justice system. To address this gap in the literature, we analyzed data from inner city, culturally diverse adolescent offenders. We chose to assess Borderline Personality using the Borderline Personality Scale of the Millon Adolescent Clinical Inventory ${ }^{[20]}$ because it is one of the few brief but well established assessment measures for measuring borderline personality features in the adolescent population. We hypothesized that adolescents with more symptoms of borderline personality would evidence greater levels of risk than their counterparts who exhibit fewer such symptoms.

\section{MATERIALS AND METHOD}

Participants: Participants were inner city, culturally diverse adolescents enrolled in two NIH-funded HIV prevention projects targeting offenders placed in juvenile detention and a court ordered treatment center. The sample approximated consecutive admissions to the program between 1998 and 2002. Entry criteria for the study included: (1) Being between ages of 13-19, (2) Having a history of drug use, (3) Being free from severe cognitive or psychiatric impairment that would have compromised their ability to complete the assessment, (4) Fulfilling assent and parental consent from parent/guardians and (5) Being fluent in spoken English.

Assessment procedures: All assessment procedures were conducted by experienced interviewers, trained to create a process sensitive to gender and cultural issues. To avoid interviewer drift and other contaminating factors, interviewers received ongoing supervision from a clinical psychologist for the duration of the study.
Subsequent to informed assent and parental consent, assessment measures were administered orally to facilitate accurate reporting, full completion and to compensate for any literacy difficulties. Assessments were administered one week after participants' admission and clearance from treatment staff that detoxification was adequately completed. This was done to minimize the effect of detoxification or withdrawal factors on test performance and to maximize the accuracy of responses. Interviewers were careful to ensure that respondents understood the meaning of each question and would repeat or elaborate on questions if a respondent showed any confusion. Interviewers were trained to adopt a non-judgmental attitude during interactions in order to establish rapport and build trust. In addition, as suggested by ${ }^{[21]}$ efforts were made to motivate participants to respond accurately, thus reducing the likelihood that reports of sexual experiences were either minimized or exaggerated. Participants were informed that their responses were confidential and would be used to help improve HIV prevention programs for other adolescents in substance abuse treatment.

Measures: Measure selection was guided by a review of the literature to determine factors of potential importance to these analyses. These variables covered the following areas: Borderline symptomatology, sex and drug risk behaviors, attitudes, knowledge and behavioral intentions.

Borderline personality scale: The Borderline Personality scale, based on 21 items from the Millon Adolescent Clinical Inventory (MACI) was used, since this scale taps the most common negative affective symptomology experienced by our sample. Other advantages of the MACI Borderline Personality (BP) Scale are that, like other MACI clinical scales, item content has been developed to operationalize formal diagnostic symptoms and the scale has demonstrated adequate reliability and validity across a wide variety of settings ${ }^{[22,24]}$. Sample items include: "I usually act quickly, without thinking"; "I do what I want without worrying about its effect on others" and "When I don't get my way, I quickly lose my temper." On the BP scale, higher scores indicate marked affective instabilities, erratic interpersonal relationships, behavioral capriciousness, impulsive hostility, fear of abandonment and self-destructive actions. For the group comparisons, MACI base-rate scores were computed.

Sex risk and drug risk variables were derived using modified versions of sexual risk assessment 
measures $^{[25,26]}$ that were adapted by Malow and colleagues $^{[27]}$. Measures included retrospective recall of numbers of sex partners, unprotected sex acts and condom use during the previous three month period (prior to being in a confined setting), as well as unprotected sex acts proximal to marijuana, alcohol and cocaine use during those previous three months. Based on focus groups and in-depth interviews, the research team has added items to the survey and modified the language to be culturally sensitive, reflecting the local terminology of the target population. In addition, we adapted a tabular format to facilitate administration and a calendar-based methodology ${ }^{[28-29]}$ to promote accurate recall. Items measuring substance use elicited the frequency of alcohol, marijuana, cocaine use during the three months prior to being in a restricted environment. This reporting period was chosen because recall has been shown to be reliable up until three months ${ }^{[30]}$.

The adolescent drug abuse diagnosis questionnaire ${ }^{[31]}$ is a structured interview recommended by the Center for Substance Abuse Treatment consensus panel $^{[32]}$ for comprehensively assessing demographic, social and psychological function among drug abusing adolescents. For the current study, we used only relevant sections to gather sociodemographic data, including age, gender, sex and level of education, living situation (with family or separate from family) and ethnicity from each subject.

Social cognitive factors: Our assessment instrument included a series of scales that reflect social cognitive factors that are related to HIV risk and risk reduction behaviors. Borrowing from models of HIV prevention such as the AIDS Risk Reduction Model ${ }^{[33]}$ and the Information, Motivation, Behavioral Skills Model ${ }^{[34]}$, we examined intentions to practice risk reducing behaviors, knowledge about HIV transmission and condom use skills. These measures are described.

The behavioral intentions scale: This scale is a 7-item measure that assesses participants' intent to take future actions to reduce HIV risk (e.g., "I will use a condom the next time I have sex"). This scale was derived by W. D. Klinkenberg (personal communication, March 1998) by simplifying an existing measure ${ }^{[35]}$ and adding an item about drinking ("I will use a condom the next time I have sex even if I've been drinking"). A subsample $(n=86)$ of the participants was used to pilot test this scale. A Cronbach's alpha of 0.94 for the behavioral intentions scale was calculated, indicating internal consistency.

Knowledge about HIV Transmission was assessed using an 18 item true/false questionnaire ${ }^{[36]}$.
Participants received one point for each correctly answered item. Sample items include: "A person can get HIV from having sex one time" and "Condoms make intercourse completely safe". Participants received one point for each correctly answered item.

Condom use skills were assessed by rating the participant's ability to properly enact nine steps in correctly placing a condom on a penile model ${ }^{[37]}$. Participants were rated for successful completion of items such as, "Opened the condom package without tearing the condom" and "Condom rolled to the base of the penile model". Scores reflect the total number of correct steps.

AIDS Risk Reduction Model: The AIDS Risk Reduction Model Questionnaire Revised ${ }^{[38]}$ was adapted and utilized to evaluate selected HIV-related risk outcomes. The ARRM-QR has been developed to reliably and validly measure HIV-specific constructs hypothesized to predict HIV risk behaviors. Constructs are defined as follows, with Chronbach's alphas calculated, ranging from 0.69-0.81: Perceived Susceptibility (perceived risk for contracting HIV), AIDS-Related Anxiety (anxiety about becoming HIV infected), sexual self-efficacy (confidence to adopt and maintain HIV preventive behaviors), personal attitudes toward condoms and sexual attitudes.

The Sexual Attitudes scale has a 5-point Likert format with response options ranging from "extremely unimportant" to "extremely important" and yields a mean score with a possible range of 1-5. The remaining scales have 4-point formats with response options ranging from "strongly disagree" to "strongly agree," and total scores ranging from 1-4. These scales have been shown to mediate HIV risk and have demonstrated validity and reliability among similar samples ${ }^{[39,40]}$.

Statistical analyses: Borderline personality scores for the sample were divided into two groups. The low borderline group included those whose scores were below 60-the cutoff score for criteria as defined on the MACI borderline scale $(n=392)$. The high borderline group consisted of those participants who scored 60$74(n=69)$, the subclinical group and $75+(n=61)$, clinical. The latter two groups were combined, as tendencies towards borderline personality are not welldefined among adolescents and both those at subclinical and clinical levels may be at risk for developing the disorder. Multivariate analysis of covariance (MANCOVAs) was used to test for group differences on sex risk behavior, substance use, HIV related attitudes and beliefs and sex risk behavior when using substances. An analysis of covariance (ANCOVA) was 
used to test for group differences on condom use skills. Significant multivariate tests were subsequently followed by paired comparisons. Adjustments were made for the following covariates in the analyses: Marijuana, cocaine and alcohol use during the previous three months, age, gender and ethnicity. These covariates were chosen based on evidence in the literature suggesting their importance in adolescent behavior. The SPSS statistical package was used for the analyses.

\section{RESULTS}

Three hundred eighty-one males and 141 females were included in these analyses (total $n=522$ ). The modal subject was a low income, ethnic or racial minority adolescent who resided in the urban inner city. The mean age of the sample was $15.74(\mathrm{SD}=1.23)$ and the average level of education was $8.72(\mathrm{SD}=1.29)$. The racial/ethnic breakdown was as follows: $35.1 \%$ were African American, 36.4\% Hispanic, 10.9\% NonHispanic white and $17.6 \%$ from other ethnic backgrounds, including Haitian American and other Caribbean heritage.

Borderline Personality symptoms group differences: Borderline personality symptoms subgroups were divided into two groups: the low borderline group included those whose scores did not meet threshold on the MACI scale $(n=392)$. The high borderline group included those whose scores were sub-clinical and clinical as defined on the MACI the borderline scale $(\mathrm{n}=130)$.

Prevalence of alcohol and other drug abuse: Participants from the low borderline personality symptoms group reported the following prevalence rates across all substances, prior to being in a restricted environment: Alcohol $\mathrm{M}=4.27$ ( $\mathrm{SD}=10.15$, range $=0$ $68)$; marijuana $\mathrm{M}=23.22(\mathrm{SD}=29.39$, range $=0-98)$; cocaine $\mathrm{M}=2.48(\mathrm{SD}=10.59$, range $=0-90)$ and intravenous drug use $\mathrm{M}=0(\mathrm{SD}=0.068$, range $=0-1)$. Participants from the high borderline personality symptoms group reported the following prevalence rates across all substances, prior to being in a restricted environment: Alcohol $\mathrm{M}=8.95$ ( $\mathrm{SD}=15.85$, range $=0$ $92)$; marijuana $\mathrm{M}=31.76(\mathrm{SD}=31.74$, range=0-101); cocaine $\mathrm{M}=4.38(\mathrm{SD}=11.92$, range $=0-89)$ and intravenous drug use $\mathrm{M}=0(\mathrm{SD}=0$, range $=0)$.

Covariate analyses: Adjustments were made for the following covariates in all MANCOVA and ANCOVA analyses: age, gender and ethnicity (Table 1). Alcohol, marijuana and cocaine use in the previous three months were also included as covariates in all analyses, with the exception of those analyses that included substance use or sex when high as the dependent variables.

Prior to their inclusion in the overall analyses, the relationship between the covariates and the borderline personality symptoms groups were assessed by use of ttest and chi-square analyses. Gender comparisons revealed no significant differences with regard to borderline group $\left(\mathrm{X}^{2}=0.04, \mathrm{p}=0.462\right)$ and no significant differences with regard to age were found $(\mathrm{t}=0.92, \mathrm{p}=0.359)$. There were no significant differences by ethnicity $\left(X^{2}=5.03, p=0.657\right)$.

HIV related attitudes and beliefs: MANCOVA analyses revealed that there were significant differences in HIV-related attitudes and beliefs by level of borderline symptoms [Wilks' $\lambda=0.958, F=2.76$, $\mathrm{p}<0.01]$ (Table 2). There were significant group differences in the following indices: Perceived susceptibility to HIV, knowledge, sex attitudes, condom attitudes, sexual response efficacy, sexual self-efficacy and behavioral intentions. Covariates including age [Wilks' $\lambda=0.923, \quad F=5.27, \quad \mathrm{p}<0.001], \quad$ gender [Wilks' $\lambda=0.947, \mathrm{~F}=3.53, \mathrm{p}=0.001$ ] and ethnicity [Wilks' $\lambda=0.956, \mathrm{~F}=2.92, \mathrm{p}<0.01]$ were significant for this analysis. Alcohol, marijuana and cocaine were not significant covariates in the analysis. A comparison of means revealed that those in the high borderline symptom group had higher perceived susceptibility to HIV, greater knowledge, less favorable sexual and condom attitudes, lower sexual self-efficacy and sexual response efficacy and less favorable behavioral intentions, than those in the low borderline symptom group.

HIV sex risk behaviors: MANCOVA results revealed that there were no significant differences in sexual risk behaviors by level of borderline symptoms.

Condom use skills: ANCOVA results revealed that there were no significant differences in condom use skills by level of borderline symptoms.

Table 1: Significant covariates in analyses testing the relationship between dependent and borderline symptom variables

\begin{tabular}{rrrllll}
\hline & \multicolumn{1}{c}{ Age } & Gender & Ethnicity & Alcohol & Marijuana & Cocaine \\
\hline$\Lambda$ & 0.923 & 0.947 & 0.956 & 0.981 & 0.982 & 0.9840 \\
& 5.270 & 3.530 & 2.920 & 1.250 & 1.160 & 1.0500 \\
& $<0.001$ & 0.001 & 0.003 & 0.268 & 0.325 & 0.3990 \\
$\Lambda$ & 0.959 & 0.969 & 0.986 & 0.960 & 0.975 & 0.9820 \\
& 3.630 & 2.730 & 1.180 & 3.540 & 2.180 & 1.5200 \\
& 0.002 & 0.013 & 0.318 & 0.002 & 0.044 & 0.170 \\
$\Lambda$ & 0.981 & 0.974 & 0.994 & N/A & N/A & N/A \\
& 3.810 & 5.290 & 1.200 & & & \\
& 0.010 & 0.001 & 0.311 & & & \\
$\Lambda$ & 0.990 & 0.992 & 0.996 & N/A & N/A & N/A
\end{tabular}


Am. J. Infect. Dis., 5 (1): 31-39, 2009

$\begin{array}{llllll}1.760 & 1.450 & 0.670 & 0.154 & 0.227 & 0.573\end{array}$

Table 2: Comparison of low Vs high borderline groups borderline symptoms and sexual risk related behaviors, substance use and attitudes for adolescents in the juvenile justice system

\begin{tabular}{|c|c|c|c|c|c|c|}
\hline & \multicolumn{2}{|c|}{$\begin{array}{l}\text { Low borderline symptoms } \\
(\mathrm{n}=392)\end{array}$} & \multicolumn{3}{|c|}{$\begin{array}{l}\text { High borderline symptoms } \\
(\mathrm{n}=130)\end{array}$} & \multirow[b]{2}{*}{$\mathrm{p}$} \\
\hline & Mean & SD & Mean & SD & $\mathrm{F}$ & \\
\hline AIDS related anxiety & 3.10 & 0.70 & 3.24 & 0.64 & 1.77 & 0.072 \\
\hline Condom attitudes & 3.28 & 0.38 & 3.18 & 0.36 & 3.38 & $<0.001$ \\
\hline Condom use skills & 4.65 & 2.28 & 4.76 & 2.13 & 1.82 & 0.063 \\
\hline Knowledge about HIV & 13.24 & 2.80 & 13.53 & 2.57 & 5.80 & $<0.001$ \\
\hline Perceived susceptibility & 2.24 & 0.62 & 2.46 & 0.62 & 2.47 & 0.009 \\
\hline Sexual self-efficacy & 3.27 & 0.49 & 3.05 & 0.54 & 4.09 & $<0.001$ \\
\hline Sexual response efficacy & 2.72 & 0.47 & 2.67 & 0.48 & 2.51 & 0.008 \\
\hline Total intentions & 22.90 & 4.88 & 22.72 & 4.40 & 3.18 & 0.001 \\
\hline Sexual attitudes & 4.36 & 0.60 & 4.20 & 0.69 & 3.52 & $<0.001$ \\
\hline Alcohol use & 4.27 & 10.15 & 8.95 & 15.85 & 6.20 & $<0.001$ \\
\hline Marijuana use & 23.22 & 29.39 & 31.76 & 31.74 & 8.12 & $<0.001$ \\
\hline Cocaine use & 2.48 & 10.59 & 4.38 & 11.92 & 3.91 & 0.001 \\
\hline Proportion of total unprotected sex acts (past 3 months) & 35.22 & 40.59 & 39.57 & 38.98 & 4.92 & $<0.001$ \\
\hline Total No. of partners (past 3 months) & 1.78 & 3.46 & 1.95 & 2.15 & 2.26 & 0.018 \\
\hline Total No. of sex acts (past 3 months) & 14.82 & 27.36 & 16.24 & 27.28 & 5.22 & $<0.001$ \\
\hline Proportion of unprotected vaginal sex (past 3 months) & 25.27 & 39.47 & 23.58 & 37.09 & 3.76 & $<0.001$ \\
\hline Proportion of unprotected oral sex-receiving (past 3 months) & 36.55 & 47.93 & 47.05 & 49.15 & 6.22 & $<0.001$ \\
\hline Proportion of unprotected oral sex-giving (past 3 months) & 18.44 & 38.62 & 23.33 & 42.05 & 3.39 & $<0.001$ \\
\hline Proportion of unprotected sex when high on alcohol & 8.76 & 27.08 & 13.99 & 32.53 & 12.90 & $<0.001$ \\
\hline Proportion of unprotected sex when high on marijuana & 15.57 & 33.36 & 18.34 & 34.65 & 8.07 & $<0.001$ \\
\hline Proportion of unprotected sex when high on cocaine & 4.52 & 20.12 & 6.45 & 23.09 & 16.61 & $<0.001$ \\
\hline
\end{tabular}

Substance use: MANCOVA results revealed that there were no significant differences in substance use by level of borderline symptoms.

Sex risk behavior when using substances: MANCOVA results revealed that there were no significant differences in sexual risk behaviors when using substances by level of borderline symptoms.

Outcome at 3 month follow-up: We followed a total of 156 of the participants originally recruited three months after completion of the intervention. Of this number, 85 were in the experimental group (75 in low borderline group, 10 in the high borderline group); 71 were in the control group (62 in the low borderline group; 9 in the high borderline group). The low borderline experimental group reported less anal sex $(\mathrm{F}(3,154)=3.72, \mathrm{p}<0.05)$, a trend suggesting lower percentage of unprotected anal $\operatorname{sex}(\mathrm{F}(3,154)=2.42$, $\mathrm{p}<0.10)$ and higher sexual self efficacy $(\mathrm{F}(3,154)=3.10$, $\mathrm{p}<0.05)$ than the high borderline experimental group at three month follow-up. The high borderline control group had higher cocaine use $(\mathrm{F}(3,154)=2.86, \mathrm{p}<0.05)$ and a trend suggesting more marijuana $(\mathrm{F}(3,154)=2.16$, $\mathrm{p}<0.10)$ and alcohol use $(\mathrm{F}(3,154)=2.46, \mathrm{p}<0.10)$ than the low borderline control group at three month followup. Interestingly, there were no differences at three month follow-up between the high borderline and low borderline experimental groups at 3 month follow-up in relation to cocaine, alcohol, or marijuana use (all ps $>0.10$ ).

\section{DISCUSSION}

This study examined the association between borderline personality symptoms and HIV risk among AOD abusing adolescents involved with the criminal justice system. Despite high levels of borderline personality features among adolescent offenders (Min et al., 2000) and studies documenting a strong association between borderline personality traits and HIV risk behavior in adults ${ }^{[41,42]}$, relatively little attention has been focused on these relationships among incarcerated adolescents.

For the purpose of these analyses, those adolescents meeting sub-clinical and those meeting clinical threshold were combined. Combining these groups made a strong distinction between those with near threshold and threshold level symptoms from those below the cutoff for criteria and this increased the validity and discriminative power of the analyses. This distinction is important as the literature shows that teens may exhibit borderline symptoms in the normal course of adolescence ${ }^{[43]}$.

These analyses revealed that there were no differences between the high and low borderline groups on age, gender or ethnicity. Adolescents in the high borderline group used more alcohol, marijuana and cocaine than those in the low borderline group, 
however these differences disappeared when demographic covariates were included in the analyses. Those in the high borderline group had higher perceived susceptibility to HIV and greater levels of knowledge about HIV, however, they had less favorable sex and condom attitudes as well as less favorable behavioral intentions. In addition, high borderline adolescents had a lower sense of sexual selfefficacy and sexual response efficacy. Thus, adolescents with higher borderline tendencies appear to be realistic in their assessments that they are at high risk of contracting HIV but also may have less confidence in their ability to adopt and maintain HIV preventive behaviors. There were no differences between groups, however, on sexual risk behaviors or condom use when the covariates were included in the analyses. These results provide support for the hypothesis that more severe borderline personality features are associated with attitudes that may lead to higher risk for contracting HIV, for example, less favorable condom attitudes, however these findings did not translate to differences between the groups on risky behaviors.

However, more detailed analyses of high borderline adolescents across control and experimental groups in a 3 month follow-up of a subset of those originally recruited participating in an HIV prevention intervention, suggested that differences in sexual risk behavior and substance use may nevertheless exist and warrant closer scrutiny. Specifically, there was evidence of more anal sex among high borderline experimental participants, which likely indicates unprotected activity and requires more dissection of the use of anal sex in the sexual behavior of high risk adolescents. Further, there was evidence of convergence between substance use, particularly cocaine and borderline symptoms. High borderline control participants reported more cocaine use than low borderline controls, while differences could not be detected in cocaine, marijuana and alcohol use between high and low borderline experimental participants as they reached 3 months post-intervention. Such results are suggestive that high borderline adolescents will become more vulnerable over time to risky behavior if risk reduction efforts are not specifically tailored to their needs in relation to substance use prevention and sexual relationships.

However, what exactly are these needs? Tailored interventions have long been a recognized priority in HIV prevention, but what this means is changing rapidly as the field adjusts to advances in neurobiological science and a paradigm resifting to biological factors and their integration into established approaches. Volkow et al. ${ }^{[4]}$ precisely discuss the impact of these advances, most particularly in visualizing the plasticity of the brain. As they make clear, neuroimaging and genotyping have provided the means to visualize function and change in the brain, yet placing demands on scientists to provide interpretive and integrative models of etiology and therapeutic intervention:

Attempts to understand and treat addiction as a purely biological or a purely environmental problem have not been very successful. Recently, important discoveries have increased our knowledge about how drugs of abuse affect biological factors such as genes, protein expression and neuronal circuits; however, much less is known about how these biological factors affect human behavior. Nor do we know much about how environmental factors affect these biological factors and how these in turn alter behavior ${ }^{[44]}$.

Thus, the resifting to biological factors is not a return to the biomedical model. Rather, it is a full-court press to catch up to the biological revelations that are proving the points made by multiple disciplines that HIV-related risk behaviors and their effects are multifactorial and ecological-in the strongest sense. Most importantly for adolescents, it means that prevention research programs, even in the urgent area of HIV/AIDS, must literally adopt a developmental perspective and methodology reflective of these principles of plasticity. This is illustrated by ${ }^{[45]}$ who try to address the developmental gap even in animal studies on the intersection of neurodevelopment and adolescent drug use.

They offer results from a research design based on adolescent rats that examines the effects of cocaine on the mesolimbic system, which may have particular salience for adolescents with borderline and antisocial characteristics. The mesolimbic pathways constitute the body's motivation and reward system and may affect the processing of novelty, interpersonal relationships and social conscience ${ }^{[46]}$ and memory formation ${ }^{[47]}$.

Donenberg et $a l .{ }^{[48]}$ have noted disjuncture in the predictive pathways of the Information-MotivationBehavior Model in their study of adolescents in psychiatric outpatient care with externalizing disorders accompanied by cognitive and emotional deficits. Catlow and Kirstein ${ }^{[49]}$ show that cocaine use during adolescence may have long-term effects on the mesolimbic system, affecting reactions to naturally occurring stimuli in an adolescent's environment long after prior use of cocaine. Even in the absence of definitive data on the role of psychiatric disorders on the mesolimbic system in relation to substance use, this kind of research only adds to the evidence of an 
exacerbating effect of psychostimulants on neurobiological mechanisms that may compound an adolescent's vulnerability in risky situations.

\section{CONCLUSION}

This small sampling of the emerging literature, in addition to the presented results of our study sample of borderline adolescents, illustrates that tailored interventions for these adolescents will depend on expanding translational research priorities from cultural to neurobiological adaptation, if their needs are to be met-and discovered in a more exacting way. Whether this effort involves neuro-or genetic technology is secondary, however, to a concentrated focus on the target population. This is demonstrated in $^{[50]}$, whose longitudinal design with adolescents in psychiatric outpatient care showed that by assessing parental hostile control in the context of whether the adolescent exhibits externalizing problems and reports positive peer influences, can provide almost $90 \%$ accuracy in identifying those reporting sexual debut by age 14 .

Volkow $^{[51]}$ suggest several routes to clinical remediation of risk for such adolescents, ranging from pharmacological to behavioral restructuring of reward structures and cognitive therapeutic interventions to strengthen the individual's frontal control. All depend, however, on an integration of neurobiological factors into behavioral prevention research. A step-wise strategy for behavioral preventionists may be to consider neurobiological factors in their pathways of action in prevention trials, including them as moderators of intervention effects to help refine the identification of phenotypes and the more "upstream" endophenotypes, the methodology of which is discussed in its fullest depth by ${ }^{[52]}$.

Results of this investigation should be viewed in light of several limitations. First, the data are crosssectional, therefore causal relationships between borderline personality symptoms and HIV risk/attitudes cannot be inferred. Second, the sample is not representative of adolescent offenders. For pragmatic and logistic reasons, it is difficult to design a study that would randomly sample adolescents from a large number of representative sites where such adolescents could be evaluated. Although we assessed adolescents at two distinctively different sites (i.e., a treatment center and a detention center), our results may not generalize to the population of adolescent offenders in the US However, the consistency of our finding with similar prior investigations supports the validity of our study. Finally, although interviewers were trained to assure youth of the confidentiality of their responses and to make them comfortable in the interview situation, all measures were based on self-reports by the youth and thus may be subject to under- or overreporting. Indeed, the interviewers reported that the youth did not appear embarrassed by sensitive questions and were forthcoming in their responses. Nevertheless, self-reports may be subject to reporting error.

Despite these limitations, our study results indicate that borderline personality symptoms may represent an important indicator of attitudes conducive to HIV transmission. Prevention efforts are much needed in this population in that sexual activity is nearly universal and contacts are risky because they are initiated early and involve multiple partners. The results also suggest the need for intensified HIV prevention efforts to focus on individuals with borderline personality features. If such efforts decrease HIV risk, this presents an important practical finding, irrespective of the nature and magnitude of the relationship of borderline personality features to HIV risk.

\section{ACKNOWLEDGEMENT}

This research was funded in part by RO1 DA11875 from NIDA and RO1 AA11752 from NIAAA.

\section{REFERENCES}

1. Castrucci, B.C. and S.L. Martin, 2002. The association between substance use and risky sexual behaviors among incarcerated adolescents. Matern. Child Health J., 6: 43-47.

2. Rotheram-Borus, M.J., Z. O'Keefe, R. Kracker and H.H. Foo, 2000. Prevention of HIV among adolescents. Prevent. Sci., 1: 15-30.

3. Jemmott, J. and L. Jemmott, 2000. HIV Behavioral Interventions for Adolescents in Community Settings. In: Handbook of HIV Prevention, Peterson, J.L. and R.J. DiClemente (Eds.). Kluwer Academic/Plenum, New York, pp: 103-127.

4. Kotchick, B.A., A. Shaffer, R. Forehand and K.S. Miller, 2001. Adolescent sexual risk behavior: A multisystem perspective. Clin. Psychol. Rev., 21: 493-519.

5. Whitaker, D.J., K.S. Miller and L.F. Clark, 2000. Reconceptualizing adolescent behavior: Beyond did they or didn't they? Family Plann. Perspect., 32: 111-117.

6. Nina, S., J.G. Johnson, P.A. Geller, R.E. Kanost and H. Zacharapoulou, 2002. Personality disorders associated with substance use among American and Greek adolescents. Adolescence, 37: 841-854.

7. Wilson, S.T., E.A. Fertuck, A. Kwitel, M.C. Stanley and B. Stanley, 2006. Impulsivity, suicidality and 
alcohol use disorders in adolescents and young adults with borderline personality disorder. Int. J. Adolescent Med. Health, 18: 189-196.

8. Marks, G., C.R.F. Bingman and T.S. Duval, 1998. Negative affect and unsafe sex in HIV-positive men. AIDS Behav., 2: 89-99.

9. Kalichman, S.C. and D. Rompa, 2001. The sexual compulsivity scale: Further development and use with HIV-positive persons. J. Personal. Assess., 76: 379-395.

10. Lavan, H. and J.G. Johnson, 2002. The association between Axis I and II psychiatric symptoms and high-risk sexual behavior during adolescence. J. Personal. Disorders, 16: 73-94.

11. Linehan, M.M., H. Schmidt, L.A. Dimeff, J.C. Craft, J. Kanterand K.A. Comtois, 1999. Dialectical behavior therapy for patients with borderline personality disorder and drug-dependence. Am. J. Addict., 8: 279-292.

12. Ross, S., H. Dermatis, P. Levounis and M. Galanter, 2003. A comparison between dually diagnosedinpatients with and without Axis II comorbidity and the relationship to treatment outcome. Am. J. Drug Alcohol Abuse, 29: 263-279.

13. Teplin, L.A., A.A. Mericle, G.M. McClelland and K.M. Abram, 2003. HIV and AIDS risk behaviors in juvenile detainees: Implications for public health policy. Am. J. Public Health, 93: 906-912.

14. Weist, M.D., D.A. Paskewitz, C.Y. Jackson and D. Jones, 1998. Self-reported delinquent behavior and psychosocial functioning in inner-city teenagers: A brief report. Child Psychiatr. Hum. Develop., 28: 241-248.

15. Gillmore, M.R., D.M. Morrison, C. Lowery and S.A. Baker, 1994. Beliefs about condoms and their association with intentions to use condoms among youths in detention. J. Adolescent Health, 15: 228-237.

16. Malow, R.M., R. McMahon, D. Cremer, J.E. Lewis and S.M. Alferi, 1997. Psychosocial predictors of HIV risk among drug abusing adolescent offenders. Psychiatr. Servic., 48: 185-187.

17. Robertson, A. and M.L. Levin, 1999. AIDS knowledge, condom attitudes and risk-taking behavior of substance-abusing juvenile offenders on probation or parole. AIDS Educ. Prevent., 11: 450-461.

18. Min, S.Y., R. Pavelsky, J. Turner, M.J. Furlong and J.M. Casas, 2000. MACI profiles of youths in a system of care. Proceeding of the Annual meeting of the American Psychological Association, Boston, MA.

19. Lavan, H. and J.G. Johnson, 2002. The association between Axis I and II psychiatric symptoms and high-risk sexual behavior during adolescence. J. Personal. Disorders, 16: 73-94.
20. Millon, T., C. Millon and R. Davis, 1993. Manual for the Millon Adolescent Clinical Inventory. National Computer Systems, Minneapolis, MN.

21. Jemmott, J.B., L.S. Jemmott and G.T. Fong, 1992. Reductions in HIV risk-associated sexual behaviors among Black male adolescents: Effects of an AIDS prevention intervention. Am. J. Public Health, 82: 372-377.

22. Davis, R.D., M. Woodward, A. Goncalves, S.E. Meagher and T. Millon, 1999. Studying Outcome in Adolescents: The Millon Adolescent Clinical Inventory and Millon Adolescent Personality Inventory. In: The Use of Psychological Testing for Treatment Planning and Outcomes Assessment, Maruish, M.E. (Ed.). 2nd Edn.

23. McCann, J.T., 1997. The MACI: Composition and Clinical Applications. In: The Millon Inventories: Clinical and Personality Assessment, Millon, T. (Ed.). Guilford, New York, pp: 363-388.

24. Millon, T. and R.D. Davis, 1993. The millon adolescent personality inventory and the millon adolescent clinical inventory. J. Counsel. Develop., 71: 570-574.

25. Gibson, D.R. and M. Young, 1994. Assessing the reliability and validity of self Reported risk behavior. In: The Context of HIV Risk Among Drug Users and Their Sexual Partners, Battjes, R.J., Z. Slobada and W.C. Grace (Eds.). NIH Publication, Rockville, MD., pp: 218-235.

26. Otto-Salaj, L.L., T.G. Heckman, L.Y. Stevenson and J.A. Kelly, 1998. Patterns, predictors and gender differences in HIV risk among severely mentally ill men and women. Commun. Mental Health J., 34: 175-190.

27. Malow, R.M., J. Devieux, T.J. Jennings, B. Lucenko and S.C. Kalichman, 2001. Substance abusing adolescents at varying levels of HIV risk: Psychosocial characteristics, drug use and sexual behavior. J. Subst. Abuse Treat., 13: 103-117.

28. Sobell, L.C. and M.B. Sobell, 1980. Convergent Validity: An Approach to Increasing Confidence in Treatment Outcome Conclusions with Alcohol and Drug Abusers.

29. Sobell, L., M. Sobell and E. Ward, 1980. In Evaluating Alcohol and Drug Abuse Treatment Effectiveness: Recent Advances. Pergamon, New York, pp: 177-183.

30. Kauth, M.R., J.S. Lawrence and J.A. Kelly, 1991. Reliability of retrospective assessments of sexual HIV risk behavior: A comparison of biweekly, three month and twelve-month self-reports. AIDS Educ. Prevent., 3: 207-214. 
31. Friedman, A.S. and A. Utada, 1989. Adolescent Drug Abuse Diagnosis. Belmont Center for Comprehensive Treatment, Philadelphia, PA.

32. McLellan, T. and R. Dembo, 1993. Improvement Protocol (TIP) Volume 3: Screening and Assessment of Alcohol and Other Drug (AOD) Abusing Adolescents (Treatment improvement protocol series). US Department of Health and Human Services, Public Health Service, Center for Substance Abuse Treatment, Washington, DC.

33. Catania, J.A., S.M. Kegeles and T.J. Coates, 1990. Towards an understanding of risk behavior: An AIDS risk reduction model. Health Educ. Q., 17, 381-399.

34. Fisher, J.D. and W.A. Fisher, 1992. Changing AIDS-risk behavior. Psychol. Bull., 111: 455-474.

35. Kalichman, S.C., K.J. Sikkema, J.A. Kelly and M. Bulto, 1995. Use of a brief behavioral skills intervention to prevent HIV infection among chronic mentally ill adults. Psychiatr. Servic., 46: 275-280.

36. Lawrence, J.S., K.W. Jefferson, E. Alleyne and T.L. Brasfield, 1995. Comparison of education versus behavioral skills training interventions in lowering sexual HIV-risk behavior of substance-dependent adolescents. J. Consult. Clin. Psychol., 63: 154-157.

37. Sorensen, J.L., J. London and E. Morales, 1991. Group Counseling to Prevent AIDS. In: Preventing AIDS in Drug Users and Their Sexual Partners, Sorensen, J., D. Wermuth, K. Gibson, J. Choi, S. Guydish and S. Batki (Eds.). Guilford, New York, USA., pp: 99-115.

38. Gibson, D.R., J. Lovelle-Drache, M.T. Young, and M. Chesney, 1992. HIV risk linked to psychopathology in IV drug users. Proceeding of the 8th International Conference on AIDS, July 1924, Center for AIDS Prevention Studies (CAPS), Amsterdam, The Netherlands, pp: 358. http://gateway.nlm.nih.gov/MeetingAbstracts/ma?f $=102200076 . \mathrm{html}$

39. Gibson, D.R. and M. Young, 1994. Assessing the Reliability and Validity of Self Reported Risk Behavior. In: The Context of HIV Risk Among Drug Users and Their Sexual Partners, Battjes, R.J., Z. Slobada and W.C. Grace (Eds.). NIH Publication, Rockville, MD., pp: 218-235.

40. Malow, R.M. and S.J. Ireland, 1996. HIV Risks Correlates Among Non-Injection Dependent Men in Treatment. AIDS Educ. Prevent., 8: 226-235.

41. Haro, G., C. Mateu, J. Martinez-Raga, J.C. Valderrama, M. Castellano and G. Cervera, 2004. The role of personality disorders on drug dependence treatment outcomes following inpatient detoxification. Eur. Psychiatr., 19: 187-192.

42. Golding, M. and D.O. Perkins, 1996. Personality disorder in HIV infection. Int. Rev. Psychiatr., 8: 253-258.

43. Chabrol, H., A. Montovany, E. Duconge, A. Kallmeyer, E. Mullet and F. Leichsenring, 2004. Factor structure of the Borderline Personality Inventory in adolescents. Eur. J. Psychol. Assess., 20: 59-65.

44. Volkow, N., J.S. Fowler and G.J. Wang, 2003. The addicted human brain: insights from imaging studies, J. Clin. Invest., 111: 1444-1451.

45. Catlow, B.J. and C.L. Kirstein, 2007. Cocaine during adolescence enhances dopamine in response to a natural reinforce. Neurotoxicol. Teratol., 29: 57-65.

46. Moll, J., F. Krueger, R. Zahn, M. Pardini, R. de Oliveira-Souza and J. Grafman, 2006. Human fronto-mesolimbic networks guide decisions about charitable donation. Proc. Natl. Acad. Sci. USA., 103pp: 15623-15628.

47. Adcock, R.A., A. Thangavel, S. Whitfield-Gabrieli, B. Knutson and D.E. John Gabrieli, 2006. Rewardmotivated learning: Mesolimbic activation precedes memory formation. Neuron, 50: 507-517.

48. Donenberg, G., R. Schwartz, E. Emerson, H. Wilson, F. Bryant and G. Coleman, 2005. Applying a Cognitive-Behavioral Model of HIV-risk to youths in psychiatric care. AIDS. Educ. Prevent., 17: 200-216.

49. Catlow, B.J. and C.L. Kirstein, 2007. Cocaine during adolescence enhances dopamine in response to a natural reinforce. Neurotoxicol. Teratol., 29: 57-65.

50. Donenberg, G., F. Bryant, H. Wilson and E. Emerson, 2003. Tracing the roots of early sexual debut among adolescents in psychiatric care. J. Am. Acad. Child Adolescent Psychiatr., 42: 594-608.

51. Volkow, N., J.S. Fowler and G.J. Wang, 2003. The addicted human brain: insights from imaging studies. J. Clin. Invest., 111: 1444-1451.

52. Eisenberg, D.T., J. Mackillop, M. Modi, J. Beauchemin, D. Dang, S.A. Lisman, J.K. Lum and D.S. Wilson, 2007. Examining impulsivity as an endophenotype using a behavioral approach: A DRD2 TaqI A and DRD4 48-bp VNTR association study. Behav. Brain Funct., 3: 1-14. 